\title{
Stiff Monatomic Gold Wires with a Spinning Zigzag Geometry
}

\author{
Daniel Sánchez-Portal, ${ }^{1}$ Emilio Artacho, ${ }^{2}$ Javier Junquera, ${ }^{2}$ Pablo Ordejón, ${ }^{3}$ Alberto García, ${ }^{4}$ and José M. Soler ${ }^{2}$ \\ ${ }^{1}$ Department of Physics and Materials Research Laboratory, University of Illinois, Urbana, Illinois 61801 \\ ${ }^{2}$ Departamento de Física de la Materia Condensada and Instituto Nicolás Cabrera, C-III, Universidad Autónoma, \\ E-28049 Madrid, Spain \\ ${ }^{3}$ Institut de Ciència de Materials de Barcelona (CSIC), Campus de la U.A.B., E-08193 Bellaterra, Barcelona, Spain \\ ${ }^{4}$ Departamento de Física Aplicada II, Universidad del Pais Vasco, Apdo. 644, E-48080 Bilbao, Spain
}

(Received 17 May 1999)

\begin{abstract}
Using first-principles density-functional calculations, gold monatomic wires are found to exhibit a zigzag shape which remains under tension, becoming linear just before breaking. At room temperature they are found to spin, which explains the extremely long apparent interatomic distances shown by electron microscopy. The zigzag structure is stable if the tension is relieved, the wire holding its chainlike shape even as a free-standing cluster. This unexpected metallic-wire stiffness stems from the transverse quantization in the wire, as shown in a simple free electron model.
\end{abstract}

PACS numbers: 68.65.+g, 71.15.Mb, 73.20.Dx, 73.40.Jn

The manipulation of matter at the atomic scale [1] is heralding a technological revolution and opening new research avenues. A spectacular achievement is the recent fabrication $[2,3]$ of monatomic chains of gold atoms, the ultimate thin wires. Metallic nanowire contacts can be created with the scanning tunneling microscope [4-6], with mechanically controllable break junctions [7], or even with simple tabletop setups [8]. The relationships between conduction, geometric, and mechanical properties have been studied by simultaneous measurements of conductance and applied force [9], by atomistic [10,11], continuous $[12,13]$, or mixed $[14,15]$ model simulations, and by first-principles calculations [16-19]. Recently, Ohnishi et al. [2] visualized nanometric gold wires by transmission electron microscopy (TEM). Surprisingly, in a stable bridge of four atoms connecting two gold tips, the atoms were spaced by 3.5-4.0 $\AA$. Later reports [20] have even increased this distance up to $\sim 5 \AA$, a value much larger than that in $\mathrm{Au}_{2}(2.5 \AA)$ and in bulk gold $(2.9 \AA)$. Gold monatomic chains with a length of four or more atoms were independently associated by Yanson et al. [3] to the last conductance plateau during stretching (close to one conductance quantum $2 e^{2} / h$ ). The histogram of these plateau lengths showed maxima at regular intervals, which may be related to the distances between gold atoms in the wire [21].

In this work we study the structure and stability of gold monatomic wires by first-principles densityfunctional calculations [22]. We use SIESTA [23], a code designed to treat large systems with local basis sets which has been already used to study gold clusters [24]. Tests were performed for $\mathrm{Au}_{2}$ and bulk gold, using both the local density approximation (LDA) [25] and the generalized gradient approximation (GGA) [26]. Core electrons were replaced by scalar-relativistic normconserving pseudopotentials [27]. Valence electrons were described with a basis set of double- $\zeta s, p$, and $d$ numerical pseudoatomic orbitals. Real- and reciprocal- space integration grids were increased until a total-energy convergence better than $2 \mathrm{meV} /$ atom was achieved. The results [28] are in very good agreement with previous calculations, using the same functionals, and with the experimental geometries and vibration frequencies [29]. The GGA improves the binding and cohesive energies, but not the geometries, which are the main focus of this work.

The wire calculations were performed for infinite monatomic chains, using periodic boundary conditions, as well as for finite wires of various lengths, either free standing or confined between small pyramidal tips. All the calculations were repeated with the LDA and the GGA, and both ferromagnetic and antiferromagnetic solutions were searched. In every case, the geometry was relaxed until the maximum forces were smaller than $10 \mathrm{meV} / \AA$ (16 pN). As an additional cross-check, some critical geometries were recalculated with a different code, using a plane wave basis set. The results will be presented in full elsewhere. In short, we have found no qualitative differences, and only very minor quantitative differences between the finite and infinite wires, between plane wave and local basis sets, and between LDA and GGA, and no magnetic solutions could be stabilized at any wire length. We present in what follows the SIESTA LDA results for the infinite wires, except where stated.

Figure 1 shows the wire geometry and the binding energy as a function of the wire length. Except when very stretched, the wire adopts a nonlinear, planar zigzag geometry, with two atoms per unit cell. Unconstrained relaxations with larger cells did not result in longer periods, nor in out-of-plane deformations. The energy shows a shallow minimum at a length of $2.32 \AA$ /atom, with a bond angle of $131^{\circ}$. The stability of this geometry was demonstrated by checking that the dynamical matrix, calculated in a cell of 16 atoms, had no negative eigenvalues. For comparison Fig. 1c shows the energy of a wire constrained to a linear geometry, which has a minimum 

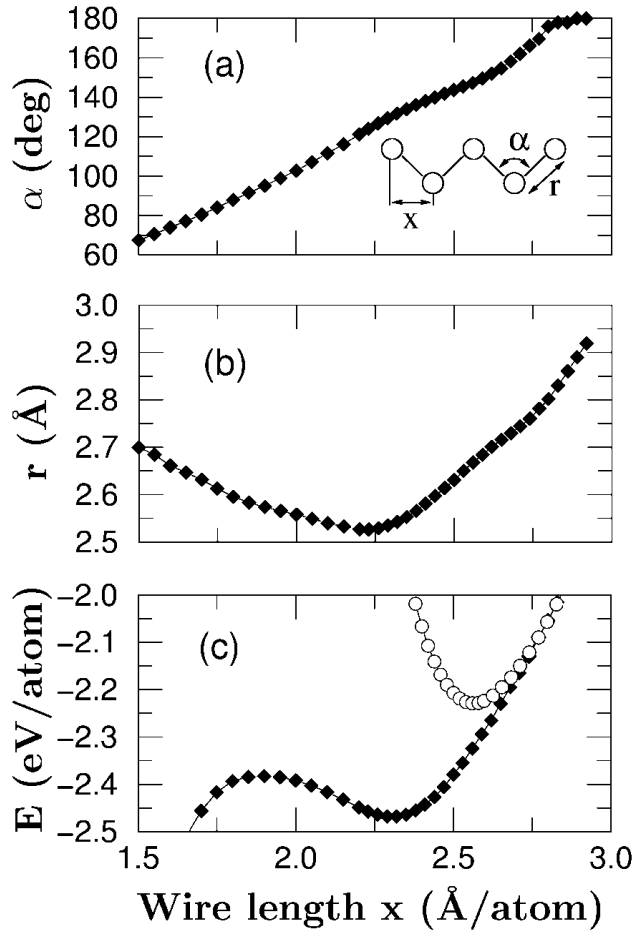

FIG. 1. First-principles, density-functional results for the bond angle $\alpha$ (a), and bond length $r$ (b) in a monatomic gold wire with zigzag geometry, as a function of its length per atom. (c) Binding energy $E$ in the zigzag (solid symbols) and linear wires (open symbols).

$0.24 \mathrm{eV} /$ atom higher, at a wire length $0.25 \AA$ longer, than in the zigzag geometry. This difference in wire length is almost entirely due to the change in bond angle, since the bond distances differ by only $0.02 \AA$ between the two minima. The bond angle increases with stretching, but the wire becomes linear only shortly before breaking.

The comparison between the band structures of the linear and zigzag wires (Fig. 2) offers some hints for understanding their relative stability. In the linear chain, the overlap between the filled $d$ states broadens the $d$ bands until they reach the Fermi level, destabilizing the wire with their associated high density of states. For the same wire length, the zigzag configuration allows a larger bond distance; that brings back the $d$ bands below the Fermi level and leaves a single $s$ band crossing it. This is consistent with the observation of a single conduction channel in the monatomic wires [3]. A Peierls dimerization instability is expected since the Fermi wave vector is at the edge of the two-atom Brillouin zone. We have observed, however, that the magnitude of this gapopening instability is negligible, only slightly noticeable just before the wire breaks, and thus playing no substantial role in the physics described here.

Although the appearance of a zigzag instability under compression may seem natural, its presence in a stretched wire is more surprising. Furthermore, its stabilization at a finite wire length is even harder to understand, since one
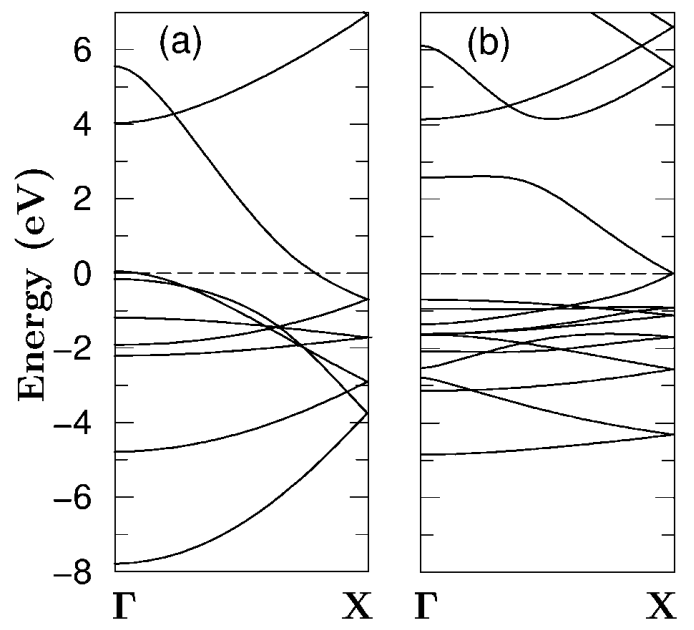

FIG. 2. Electronic band structure of the linear (a) and zigzag (b) wires for a length of $2.32 \AA$ /atom. The linear-wire bands have been folded onto a two-atom Brillouin zone to facilitate the comparison. The energies are relative to the Fermi level.

would expect the wire to collapse into a compact, highcoordination structure typical of metals. However, we find that even free-standing clusters of four or eight atoms (the sizes calculated) are also stable with a zigzag chain structure. A possible chemical-bond directionality effect was first considered, but the electron density showed a clear homogenization tendency, with a net depletion in the interatomic regions. A zigzag stabilization due to covalent bonding was thus discarded. The origin appears very naturally, however, in a delocalized electron picture, from the transverse quantization of the electron states. To see this, we model the wire as an infinite straight tube with a rectangular section $b \times c$ and a length $a$ per atom (or per $s$ electron). The difference between $b$ and $c$ accounts for the extra width due to the zigzag (see the inset in Fig. 3). Consistently with the standard jellium model [13], we assume a fixed

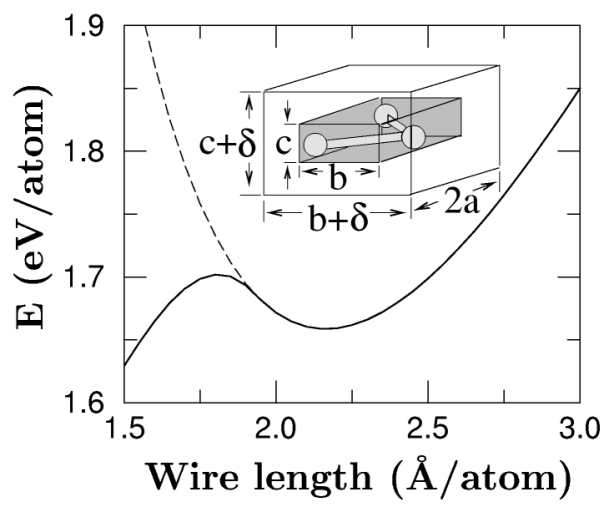

FIG. 3. Energy versus wire length for a simple model of the wire, considered as a free-electron tube of fixed volume, as shown in the inset, with $a_{0}=\sqrt{a b}=3 \AA$ and $\delta=2 \AA$. Dashed and solid lines correspond to allowing one or two occupied bands, respectively. 
volume per atom $a b c$, but we allow a larger "box" section $(b+\delta) \times(c+\delta)$ to account for an electron "spillage" $\delta / 2$ out of each jellium edge [30]. Accepting from the $a b$ initio calculation that the zigzag is planar, we also fix its thickness $c$ or, equivalently, $a_{0}=\sqrt{a b}$. The resulting free-electron energy is shown in Fig. 3 as a function of the wire length $a$, for reasonable values of $a_{0}$ and $\delta$. With a single occupied band, the compromise between the transversal and longitudinal kinetic energies results in a single minimum (dashed line). Including the second band, which becomes partially occupied at somewhat shorter lengths, allows the energy to decrease again (solid line), reproducing all the qualitative features observed in the $a b$ initio curve, such as the positions of the maximum, the minimum, and the point at which the second band crosses the Fermi level (1.83 $\AA$ /atom). The basic physics that this model illustrates is the higher stability of certain wire sections, due to the transverse quantization of the delocalized electron states [13]. This shell structure effect, which has been recently observed for sodium wires [31], is similar to the so-called magic numbers (particularly stable sizes) of small metal clusters [32]. The zigzag shape is a particular realization of these stable sections for the monatomic gold wires.

In agreement with previous ab initio calculations [19], we find that the wire becomes unstable and breaks spontaneously when pulled by a force of more than $2.2 \mathrm{nN}$, i.e., beyond a maximum length of $2.9 \AA$ /atom, much shorter than that apparently observed in stable wires [2]. We offer here an explanation for this puzzling discrepancy, based on the predicted zigzag geometry: if the actual wires observed have an odd number of atoms, with those at the extremes fixed by the contacts, the oddnumbered atoms would stay almost fixed on the same axis, while the even-numbered ones could rotate rapidly around that axis, offering a fuzzy image that could be missed by the TEM. We have calculated the relaxed geometry and the rotation energy barrier for a seven-atom wire suspended between two pyramidal tips made of three atoms in contact with the wire, plus six atoms below. The latter are kept fixed in their bulk positions. The resulting stable geometry is almost equal to that of the infinite wire, and the rotation barrier is only $60 \mathrm{meV}$ for the entire wire. The effect is illustrated in Fig. 4, where we show the electron density averaged over rotated configurations. Although not directly comparable to a TEM image, it can indeed be qualitatively appreciated that the odd-numbered atoms appear much sharper than the even-numbered ones, giving the impression of a four-atom wire with a large interatomic separation, similar to that observed experimentally. The intensity of a given atom is, roughly, inversely proportional to its rotation radius and, for the structure in Fig. $4, R_{\text {even }} / R_{\text {odd }} \sim 5$. From the energy barrier obtained, we estimate that the thermal rotation would slow down to the millisecond scale, allowing the zigzag visualization, only for temperatures below $\sim 40 \mathrm{~K}$.

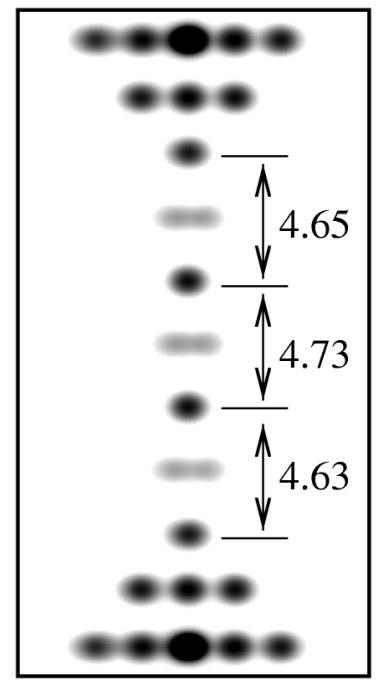

FIG. 4. Calculated electron density of a seven-atom wire suspended between two small pyramidal tips, averaged over all rotation angles around the wire axis, with a Boltzmann factor at $T=300 \mathrm{~K}$. The numbers are relaxed distances in angstroms.

In a wire with an even number of atoms, all of them would rotate with an appreciable radius, making their visualization more difficult.

Figure 5(a) shows the calculated transversal and longitudinal phonon frequencies at $\Gamma$, for the zigzag wire, as a function of its length. Negative values indicate modes with imaginary frequency, implying the breaking of the unstable wire. At the wire's equilibrium length $(2.32 \AA /$ atom $)$, the $\Gamma$-point frequencies are 113 and $219 \mathrm{~cm}^{-1}$, for the transversal and longitudinal modes, respectively. These are quite larger than the bulk phonon frequencies, but comparable to those of the dimer (the
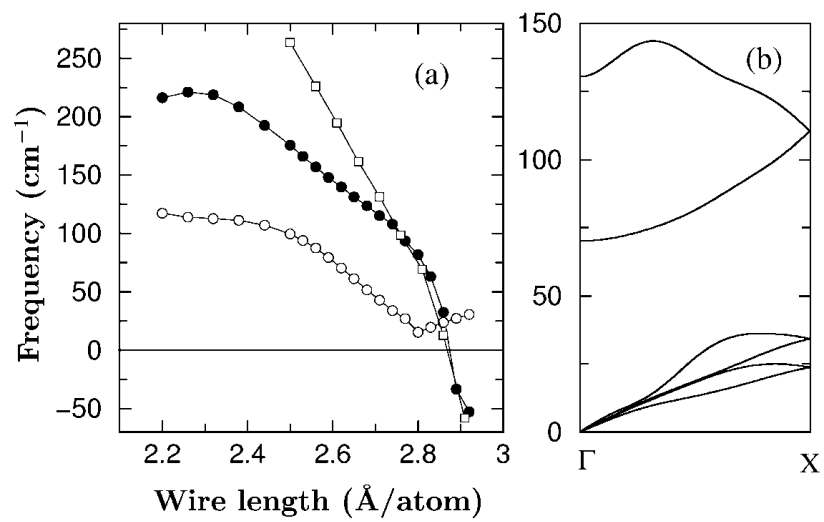

FIG. 5. (a) Wire-length dependence of the optical phonon frequencies of a zigzag wire at $\Gamma$, calculated using the frozen-phonon method. Solid and open circles stand for the longitudinal and transversal modes respectively. For comparison, we also show the longitudinal phonon frequency at $X$ (which folds to $\Gamma$ in a two-atom cell) for a wire constrained to be linear (squares). (b) Phonon dispersion curves for a zigzag wire of $2.62 \AA$ /atom length. 
wire interatomic distance is only slightly larger than that in $\mathrm{Au}_{2}$ ). Figure 5(b) shows the phonon dispersion relations for a wire length of $2.62 \AA /$ atom, obtained from the full dynamical matrix in a supercell of sixteen atoms, calculated with finite differences [33]. We hope that the comparison of the results in Fig. 5 with those of point contact spectroscopy experiments [34] will help to confirm our predicted zigzag distortion.

We thank C. Balbás, N. García, J. Kohanoff, J. J. Sáenz, J. A. Torres, and E. Tosatti for useful discussions, and N. Agraït and G. Rubio also for making their results available to us prior to publication. D. S.P. is grateful to R. M. Martin for advice and support. This work was supported by Grants No. DGES PB95-0202, No. DOE 8371494, and No. DEFG 02/96/ER 45439.

[1] D. M. Eigler and E. K. Schweizer, Nature (London) 344, 524 (1990).

[2] H. Ohnishi, Y. Kondo, and K. Takayanagi, Nature (London) 395, 780 (1998).

[3] A.I. Yanson, G. Rubio Bollinger, H.E. van den Brom, N. Agraït, and J.M. van Ruitenbeek, Nature (London) 395, 783 (1998).

[4] N. Agraït, J. G. Rodrigo, and S. Vieira, Phys. Rev. B 47, 12345 (1993); N. Agraït, G. Rubio, and S. Vieira, Phys. Rev. Lett. 74, 3995 (1995).

[5] J. I. Pascual et al., Phys. Rev. Lett. 71, 1852 (1993); Science 267, 1793 (1995).

[6] L. Olesen et al., Phys. Rev. Lett. 72, 2251 (1994).

[7] C. J. Muller, J. M. van Ruitenbeek, and L.J. de Jongh, Phys. Rev. Lett. 69, 140 (1992); J. M. Krans et al., Nature (London) 375, 767 (1995); E. Scheer et al., Nature (London) 394, 154 (1998).

[8] J. L. Costa-Krämer, N. García, P. Gacía-Mochales, and P. A. Serena, Surf. Sci. 342, L1144 (1995).

[9] G. Rubio, N. Agraït, and S. Vieira, Phys. Rev. Lett. 76, 2302 (1996).

[10] U. Landman, W. D. Luedtke, N. A. Burnham, and R. J. Colton, Science 248, 454 (1990).

[11] T. N. Todorov and A. P. Sutton, Phys. Rev. Lett. 70, 2138 (1993).

[12] J. A. Torres and J. J. Sáenz, Phys. Rev. Lett. 77, 2245 (1996).

[13] C. A. Stafford, D. Baeriswyl, and J. Bürki, Phys. Rev. Lett. 79, 2863 (1997); J. M. van Ruitenbeek, M. H. Devoret, D. Esteve, and C. Urbina, Phys. Rev. B 56, 12566 (1997);
C. Yannouleas and U. Landman, J. Phys. Chem. B 101, 5780 (1997); S. Blom et al., Phys. Rev. B 57, 8830 (1998).

[14] N. D. Lang, Phys. Rev. Lett. 79, 1357 (1997).

[15] C. C. Wan et al., Appl. Phys. Lett. 71, 419 (1997).

[16] R. N. Barnet and U. Landman, Nature (London) 87, 788 (1997).

[17] D. Sánchez-Portal et al., Phys. Rev. Lett. 79, 4198 (1997).

[18] A. Nakamura, M. Brandbyge, L. B. Hansen, and K. W. Jacobsen, Phys. Rev. Lett. 82, 1538 (1999).

[19] J. A. Torres et al., Surf. Sci. 426, L441 (1999).

[20] Y. Kondo and K. Takayanagi, Bull. Am. Phys. Soc. 44, 312 (1999).

[21] A recalibration of the STM setup yields $2.7 \pm$ $0.3 \AA$ /atom for the wire breaking lengths, smaller than that initially reported in Ref. [3], and in good agreement with our result and with that of Ref. [19] [G. Rubio Bollinger (private communication)].

[22] W. Kohn and L. J. Sham, Phys. Rev. 140, 1133 (1965).

[23] P. Ordejón, E. Artacho, and J.M. Soler, Phys. Rev. B 53, R10 441 (1996); D. Sánchez-Portal, P. Ordejón, E. Artacho, and J. M. Soler, Int. J. Quantum Chem. 65, 453 (1997); Phys. Status Solidi (b) 215, 809 (1999).

[24] I. L. Garzón et al., Phys. Rev. Lett. 81, 1600 (1998).

[25] J. P. Perdew and A. Zunger, Phys. Rev. B 23, 5075 (1981).

[26] J.P. Perdew, K. Burke, and M. Ernzerhof, Phys. Rev. Lett. 77, 3865 (1996).

[27] N. Troullier and J.L. Martins, Phys. Rev. B 43, 1993 (1991).

[28] In the LDA, we obtain, for the gold dimer, a bond length $l=2.51 \AA$, a vibration frequency $\nu=190 \mathrm{~cm}^{-1}$, and a binding energy $D=3.18 \mathrm{eV}$. For the bulk fcc crystal, the calculated nearest-neighbor distance, bulk modulus, and cohesive energy are $d=2.91 \AA, B=194 \mathrm{GPa}$, and $E_{c}=4.55 \mathrm{eV}$, respectively. In the GGA, the results are $l=2.57 \AA, \nu=171 \mathrm{~cm}^{-1}, D=2.72 \mathrm{eV}, d=2.98 \AA$, $B=137 \mathrm{GPa}$, and $E_{c}=3.37 \mathrm{eV}$. The experimental values are $l=2.47 \AA, \nu=191 \mathrm{~cm}^{-1}, D=2.29 \mathrm{eV}$, $d=2.87 \AA, B=172 \mathrm{GPa}$, and $E_{c}=3.78 \mathrm{eV}$.

[29] O. Häberlen, S.C. Chung, and N. Rösch, Int. J. Quantum Chem. Quantum Chem. Symp. 28, 595 (1994); B. D. Yu and M. Scheffler, Phys. Rev. B 56, R15 569 (1997).

[30] A. García-Martin, J. A. Torres, and J.J. Sáenz, Phys. Rev. B 54, 13448 (1996).

[31] A.I. Yanson, I. K. Yanson, and J. M. van Ruitenbeek, Nature (London) 400, 144 (1999).

[32] W.D. Knight et al., Phys. Rev. Lett. 52, 2141 (1984); W. A. de Heer, Rev. Mod. Phys. 65, 611 (1993).

[33] P. Ordejón, D. A. Drabold, R. M. Martin, and S. Itoh, Phys. Rev. Lett. 75, 1324 (1995).

[34] C. Untiedt, G. Rubio, S. Vieira, and N. Agraït, Phys. Rev. B, 56, 2154 (1997), and references therein. 